\title{
Control of Centerline Segregation in Slab Casting
}

\author{
Mihály Réger ${ }^{1}$, Balázs Verö ${ }^{2}$, Róbert Józsa ${ }^{3}$ \\ 'Óbuda University, Bánki Donát Faculty of Mechanical Engineering, Bécsi út \\ 96/b, 1034 Budapest, Hungary, reger.mihaly@bgk.uni-obuda.hu \\ ${ }^{2}$ Bay Zoltán Nonprofit Ltd. for Applied Research, Fehérvári út 130, 1116 \\ Budapest, Hungary, vero@bzaka.hu
}

${ }^{3}$ ISD Dunaferr Co. Ltd., Vasmü tér 1-3, 2400 Dunaújváros, Hungary, jozsa.robert@isd-dunaferr.hu

\begin{abstract}
A complex mathematical model characterizing the centerline segregation level in the midregion of continuously cast slabs was developed. The basic heat transfer and solidification model connected to the semi-empirical liquid feeding model (LMI - Liquid Motion Intensity model) gives the possibility to estimate the centerline segregation parameters of slab cast under industrial circumstances. Solid shell deformation changes the volume of the space available for the liquid inside the slab and hereby also changes the conditions of liquid supply. In modelling slab casting in practical industrial cases the deformation of the solid shell cannot be ignored, especially from the point of view of centerline segregation formation. From this aspect, the most important effects resulting in deformation of the solid shell are as follows: shrinkage of the solid shell due to solidification and cooling; setting of the supporting rolls along the length of the casting machine i.e. decreasing the roll gaps as a function of cast length; bulging of the solid shell between successive supporting rolls; positioning errors and wear of rolls; eccentricity of individual rolls; etc. The critical parameter to describe the inhomogeneity in the center area of slabs is the porosity level in the mushy region. As a result of calculations performed by the model, ISD Dunaferr Co. Ltd. has changed the strategy of supporting roll settings in their continuous casting. After the modification had been implemented on casting machines, the quality problems due to centerline segregation of slabs decreased to a great extent.
\end{abstract}

Keywords: slab casting; centerline segregation; porosity; deformation of solid shell; mushy; permeability; pressure drop 


\section{Introduction}

The continuous casting of slabs is aimed at producing a product with a proper chemical composition, geometry and surface quality, without any or a minimum acceptable level of external and internal defects. One of the most unpredictable defects of the slabs is centerline segregation, which has a negative effect on further processing of the slabs and hence on the possible uses of the final product.

The solidification of continuously cast products is accompanied by the volume changes of shrinkage and deformations. In order to compensate for the volume changes, the gaps between the supporting rolls decrease along the casting machine as a function of distance from the meniscus level. The proper compensation is important mainly in the last third of the solidification, where the center area of the strand has a two-phase mushy structure (mushy area of the slab). In case of improper correspondence between volume change and roll setting, the melt is forced to flow in the mushy area. If the liquid supply is insufficient to compensate volume changes, then discontinuities or inner porosity will develop, typically accompanied with macrosegregation. This phenomenon is the centerline segregation. The required liquid supply is provided by melt flow due to ferrostatic pressure. The liquid flow in the mushy area of the strand is impeded by the network of solid dendrites, and hence ferrostatic pressure decreases and liquid supply becomes uncertain. The reduction itself and the reduction rate of ferrostatic pressure play a key role in porosity formation. In order to investigate porosity formation, it is necessary to learn about the volume changes inside the strand. The Liquid Motion Intensity (LMI) model provides these kind of data ${ }^{1,2)}$. The suitability of the LMI model for the estimation of centerline segregation level has been demonstrated in previous research ${ }^{3,4)}$.

\section{Nature of the Centerline Segregation}

Centerline segregation of slabs relates partly to macrosegregation and partly to the shrinkage of melt, the formation of small shrinkage holes and, occasionally, the formation of inclusions ${ }^{5-8)}$. In continuous casting centerline segregation develops in the middle part of the slab due to solidification and transformation processes, to fluid flow and also to constrained liquid supply, which is necessary for solidification shrinkage compensation. Only enriched melt is present between solid dendrite trunks. Any effect that enhances fluid flow (i.e. cooling conditions, setting of the supporting rolls, bulging between successive rolls, etc.) necessarily results in the flow of the enriched melt, i.e. macrosegregation will form. The possibility of sufficient liquid supply in the mushy area decreases depending on the ratio of solid phase. At the same time the permeability of zigzag channels between dendrite arms also decreases. Lessening the possibility of liquid supply 
inevitably leads to the formation of shrinkage holes and porosity, which is also typical of centerline segregation formation. In Fig. 1, photographs taken by a scanning electron microscope, shows details of the rupture surface in the slabs mid-region. Relatively fine dendrites and highly fragmented channels of fluid flow can be identified in these pictures.

The macrosegregation part of centerline segregation can be characterized by the segregation ratio of individual elements ${ }^{5}$. Porosity can be measured by metallographic, ultrasound or density measurement methods ${ }^{5-7)}$. In everyday industrial practice, steel producers prefer to apply cheap, fast and automated methods to characterize centerline segregation. The two most common methods are: comparison with standard images and measurement of the amount of shrinkage holes by image analysis. The latter one produces a relatively well quantifiable result. Because of the connection between the amount of shrinkage holes and the level of macrosegregation ${ }^{7)}$, the application of image analysis method and characterization of centerline segregation of the slabs by the porosity level is widely accepted by industry.
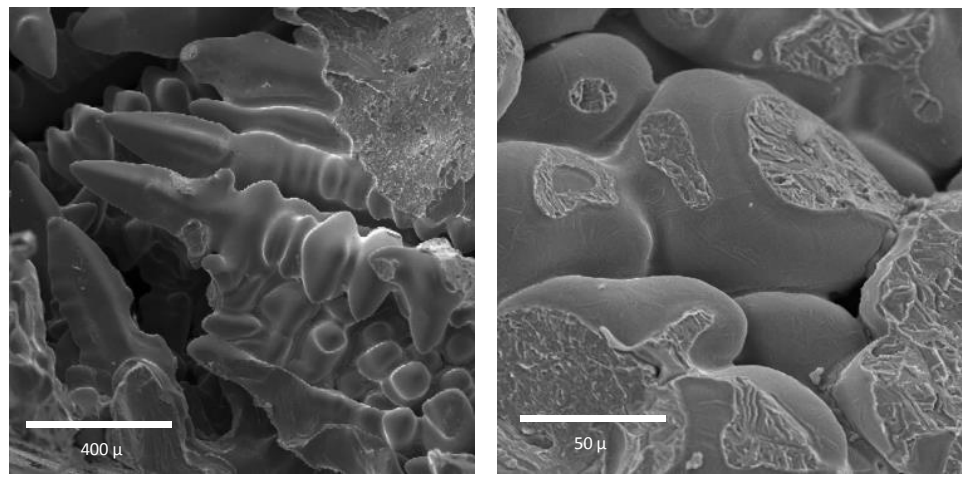

Figure 1

Shrinkage holes in the mid part of continuously cast slab

Despite the high ratio of plastic deformation, strips hot rolled from slabs with centerline segregation contain, in a modified form, the consequences of this type of inner defect ${ }^{9}$. As a result of inheritance, the middle part of the strip has a chemical composition (and structure) different from the average, which results in differing properties in the mid section of strip. The thickness of the defected part in the strip depends on the extent of plastic deformation. In general, the lower the thickness of the strip (i.e. the higher the amount of plastic deformation), the thinner the defect is in the strip. Accordingly, centerline segregation can cause problems in particular in the further processing of heavy plates (during cutting, drilling, welding, etc.).

Experience shows that the unfavorable properties of strips due to centerline segregation cannot be improved at a later stage and the level of macrosegragation 
cannot be reduced significantly ${ }^{10,11)}$. This is explained, initially, by the cross effects of diffusion processes of individual enriched elements (e.g. the local manganese content affects the diffusion of carbon).

It follows that the centerline segregation level can only be controlled during the solidification process. The resultant acceptable centerline segregation level for the users must hence be ensured by the application of a proper continuous casting technology.

\section{Characteristics of the Mushy Area of the Slab}

A sketch of the structure of a cast slab is shown in Fig. 2. If a vertical type casting machine is used, during casting the slab is in a vertical position. In curved casting machines, on the other hand, solidification starts in a vertical position but is completed in a horizontal position. In the first stage of solidification the dendrites growing from both sides do not reach each other and the center part of the strand contains pure liquid steel. The fluid flow in the upper part of the strand is mainly controlled by the inlet of steel from a submerged entry nozzle, by differences in density (thermal or solutal) and also by the deformation of the solid. Deformations of the solid shell originate from shrinkage (solidification, cooling and re-heating of the shell, allotropic transformations), from bending of the strand, from setting of the roll gaps and from bulging between successive rolls. The liquid flow and liquid supply (in the casting direction and perpendicular to the casting direction between dendrites) are supposed to be unlimited because only liquid can be found in the center part of the strand.

Depending on the casting parameters and on the composition of steel at a given distance from the meniscus level, the solidification fronts (liquidus fronts) growing from both sides touch each other. Therefore, (for the sake of simplicity, columnar solidification of the dendrites is supposed) the tips of the dendrites reach each other. In the case of slab casting in a curved caster, this occurs at about 12-15 $\mathrm{m}$ from the meniscus level in the unbent zone or after it. In a vertical casting machine the beginning of the mushy area is about 5-7 $\mathrm{m}$ from meniscus because of the constrained metallurgical length. 


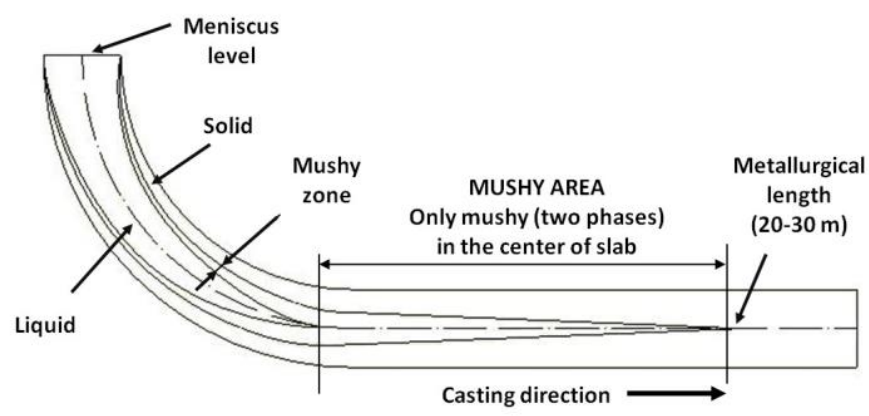

Figure 2

Sketch of inner structure of cast slab

At the beginning of the mushy area, the temperature of dendrite tips that meet each other is approximately equal to liquidus. From the point of view of liquid flow, a fundamentally different situation starts here because liquid supply in the casting direction must be realized through the zigzag and highly fragmented channels of the solid dendrite structure. The amount of liquid necessary in the mushy area is determined by the deformations in the mushy area and of the solid shell (mainly the shrinkage of the solid shell, setting of the supporting roll gaps, bulging). The roll gap in this case includes the prescribed roll gap setting, the errors of this setting, the wear and eccentricity of individual rolls.

Realization of the necessary amount of liquid supply through the fragmented tunnel system of dendrite arms depends on the pressure conditions that have developed in the liquid of the mushy area. The ferrostatic pressure of the liquid is determined by the height of liquid steel and by the pressure drop due to the constrained melt flow through the porous medium. The pressure drop depends on the volumetric flow rate and on the permeability in the mushy area. The permeability is the function of liquid ratio and the characteristics of the primary dendrite structure.

In the last stage of solidification the liquid supply must be realized by liquid flow through the mushy area. The length of the mushy area is between $3-10 \mathrm{~m}$, depending on the structure of the casting machine and the casting technology (see Fig. 2). Ferrostatic pressure within this distance can decrease, to such an extent, that it is no longer sufficient to produce the required melt supply.

According to preliminary calculations, pressure drop is very low above the mushy area. This has no effect on the liquid supply in the casting direction or perpendicular to the casting direction of the mushy zone. However, in the mushy area, in casting direction, the melt supply is likely to be hindered. It should be noted that in the last stage of solidification the pressure conditions and liquid supply also affected by the formation of porosity (melt sucking) and the pressure of gases released inside the pores. 


\section{Pressure Drop in the Mushy Area}

Decrease in the ferrostatic pressure in the interdendrite channels within the mushy area, can be estimated by the Darcy law:

$$
Q=\frac{-K A}{\mu} \frac{\Delta P}{L}
$$

where $Q$ the volumetric flow rate $\left(\mathrm{m}^{3} / \mathrm{s}\right), A$ the cross sectional area $\left(\mathrm{m}^{2}\right), \Delta P$ the pressure drop (Pa), $\mu$ viscosity of the melt (Pas), $L$ length of the section under investigation $(\mathrm{m}), K$ permeability $\left(\mathrm{m}^{2}\right)$.

In order to estimate the pressure drop, the volumetric flow rate along the mushy area must be known. Volumetric flow rate in the mushy area of the strand depends on the space available for liquid inside the strand. In the calculation of this space, shrinkage during solidification and cooling (chemical composition of steel, cooling conditions, etc.), and deformations of the strand (settings of the supporting rolls, bulging) must be taken into account. The volumetric flow rate can be calculated by the Liquid Motion Intensity (LMI $)^{1-4)}$ model, taking into consideration also all the above mentioned and important deformation effects.

\section{Main Characteristics of the LMI Model}

The Liquid Motion Intensity ${ }^{1-4)}$ (LMI) 2D model is used for the calculation of volume changes inside the strand under steady and non-steady casting conditions within the longitudinal cross section of the slab. The main task of the model is to define volumetric flow rate function $(Q)$ inside the strand, taking into account the effect of chemical composition of the steel, the steel casting technology and the casting machine parameters as well. The main idea of the model is that the amount of liquid entering or exiting from a volume section (slice) of a strand at a given distance from meniscus can be calculated by taking into account the effect of composition, casting technology and casting machine. The amount of melt moving between the slices can be summarized along the whole strand or along the mushy area and from this the amount of melt flow and the rate of the flow can be defined. A detailed description of the model principles and of the simplifications applied can be found in earlier publications ${ }^{1-4)}$. The following deformations of the strand can be taken into account in the model:

- Shrinkage of solidification

- Shrinkage of solid shell because of temperature changes

- Shrinkage of solid due to transformations 
- $\quad$ Nominal roll gap settings along the casting machine

- Real roll gaps along the casting machine (roll checker data, if available)

- Eccentricities of supporting rolls (if data are available)

- Bulging of the solid shell between supporting rolls (calculated or measured data, if available).

In order to define the volume changes inside the slab, thermal and solidification modeling of slab casting is necessary. Thermal and solidification data are provided for the LMI model by the following software:

- IDS (Interdendritic solidification) - calculation of composition and temperature dependent material data

- TEMPSIMU (Temperature simulation of CC) - 3D temperature and solidification model of cast strand for steady and non-steady state casting conditions

- $\quad$ BOS (Bulging of slab) - determination of bulging between successive rolls

All the above software was developed and tested by the Laboratory of Metallurgy, Helsinki University of Technology (today: Aalto University).

\section{Permeability of the Mushy Area}

The permeability of isotropic porous medium is typically described by using the Kozeny-Carman equation ${ }^{12)}$ :

$$
K=\frac{1}{k S_{V}} \frac{g_{L}{ }^{3}}{\left(1-g_{L}\right)^{2}}
$$

where $k$ Kozeny constant (supposed to be $5^{12)}$ ), $S_{V}$ the solid/liquid surface in unity volume $\left(\mathrm{m}^{2}\right), g_{L}$ ratio of liquid. The equiaxed dendrite structure can be considered to be isotropic porous medium.

A number of experiments have been performed to define the permeability in case of non-isotropic interdendrite fluid flow (e.g. solidification with columnar structure). The authors published their results in the form of empirical equations ${ }^{13-}$ ${ }^{15)}$. In these calculations the primary and secondary dendrite arm spacing and the direction of flow compared to primary dendrite arm are also taken into consideration. The permeability parallel to primary arms and perpendicular to primary arms are different.

If liquid ratio is not too high, the results of the different models give similar results. In general, the liquid in the mushy area is typically between a 0 and 0.6 
ratio. For the calculation of permeability in the mushy area the equation published by Bhat et. al. ${ }^{13)}$ was considered:

$$
\begin{aligned}
& K_{P}=3.75 \cdot 10^{-4} d_{1}^{2} g_{L}^{2} \quad\left(\mathrm{~g}_{\mathrm{L}}<=0,65\right) \\
& K_{N}=1.09 \cdot 10^{-3} d_{1}^{2} g_{L}^{3,32} \quad\left(\mathrm{~g}_{\mathrm{L}}<=0,65\right)
\end{aligned}
$$

where $K_{P}$ permeability parallel to primary arms $\left(\mathrm{m}^{2}\right), K_{N}$ permeability perpendicular to the growth direction of primary arms $\left(\mathrm{m}^{2}\right), d_{l}$ primary dendrite arm spacing $(\mathrm{m})$. In this model the secondary spacing is taken into account through the correlation of primary and secondary spacing.

\section{Application for Continuous Casting}

The ferrostatic pressure drop in the mushy area and its consequences are presented in a practical example of casting on a curved machine. In order to demonstrate the practical applicability, the calculation was performed by supposing two different roll setting strategies. In the first case there is no change in roll gaps along the casting machine (constant setting). In the second case a roll setting applied in the industrial practice was used (prescribed setting used by the steel producer). Although the first case has no practical relevance, the difference between the two cases highlights the extremely important role of a precise roll setting from the viewpoint of centerline segregation. The roll gap data applied in the calculations are shown in Fig. 3a as a function of distance from the meniscus level.

\subsection{Volumetric Liquid Flow Rate Function}

Liquid flow conditions in the mushy area are presented in Fig. $3 \mathrm{~b}$ for both roll setting strategies. In this casting case the pool lengths are: $15.7 \mathrm{~m}$ (for the liquidus temperature) and $23.8 \mathrm{~m}$ (for the solidus temperature). Hence the mushy area (see Fig. 2) starts at $15.7 \mathrm{~m}$ and ends at $23.8 \mathrm{~m}$; its length is $8.1 \mathrm{~m}$. Between meniscus and $15.7 \mathrm{~m}$, the mid part of the strand contains homogeneous liquid and the liquid supply is not hindered ( $Q$ is not calculated for this part of the strand). After solidification has been completed (the distance from meniscus is over $23.8 \mathrm{~m}$ ), there is no more liquid in the slab, hence $Q=0$. In the mushy area, between 15.7 and $23.8 \mathrm{~m}$ for compensation of volume changes, liquid steel flows into the cross sections of the mushy area. By the application of the LMI model (summarizing the liquid necessary for each volumetric slice), the liquid flow rate function in Fig. $3 \mathrm{~b}$ can be calculated. The function gives the amount of necessary flow rate of liquid for each cross section in the mushy area. At the beginning of the mushy area (at $15.7 \mathrm{~m}$ ) the value of the function indicates the amount of necessary liquid that should enter the mushy area from the direction of meniscus in order to maintain 
the solidification without formation of discontinuity. By definition, the positive value of the function indicates the flow in the casting direction. Fig. $3 \mathrm{~b}$ shows the volumetric liquid flow rate function for both roll settings (Fig. 3a).

From the viewpoint of centerline segregation it is desirable that flow rate be close to zero. The flow rate function (Fig. 3b) is basically determined by the setting strategy of the supporting rolls. The figure also indicates that if the roll setting is fine tuned, the flow rate can be further reduced.

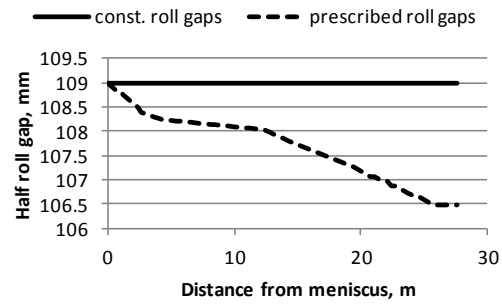

a/

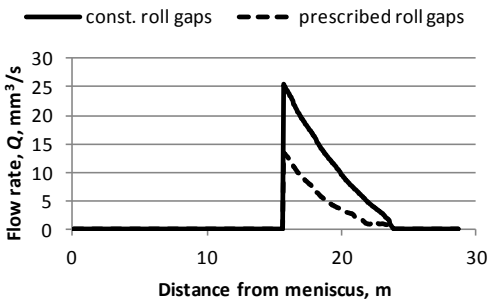

b/

Figure 3

Flow rate necessary for solidification without discontinuities (b/)

for two different settings of the supporting rolls $(\mathrm{a} /)$

The volumetric flow rate function indicates the harmony between the steel composition to be cast, the casting technology and the casting machine. The better the harmony, the closer the function approaches zero. The flow rate function is applicable for the complex evaluation of a casting case from the point of view of probability of centerline segregation formation.

\subsection{Pressure Drop and Porosity Function}

If the volumetric flow rate function, the permeability and the geometric and microstructure parameters of the dendrite structure are known, the ferrostatic pressure drop in the mushy area can be estimated. For the explanation of results let us consider the diagram in Fig. 4 showing the ferrostatic pressure drop in the mid part of the slab in the mushy area. The two vertical lines indicate the beginning and the end of the mushy area. The dotted line represents the ferrostatic pressure evolving as a result of difference in height between the actual position and the meniscus level (curved machine). The thin line describes the ferrostatic pressure drop caused by the liquid flow in a porous medium; it can be calculated by Eq. (1). The final ferrostatic pressure (sum of the original ferrostatic pressure and of the pressure drop) in the liquid is represented by the thick dashed line. As a result of pressure drop in the mushy area, the final pressure decreases drastically (depending on casting and structural parameters) and can even reach zero value. 
The distance from the meniscus level at which zero pressure was reached is the Zero Liquid Supply (ZLS) value. The ZLS point divides the mushy area into two parts. From the ZLS to the direction of meniscus (on the left), sufficient liquid supply is provided by the ferrostatic pressure. When the ferrostatic pressure reaches zero, there is no more pressure that would force the melt to fill the volume changes developed by shrinkage and deformation. In this range, melt supply is not necessarily provided. Cavities formed during solidification can exert a sucking influence on the surrounding melt but its rate and efficiency are rather uncertain in the highly fragmented channel network between solid dendrites. Actual pressure is also affected by gases released inside the cavities. It is assumed in the calculations that after the ZLS point has been reached, there is no further possibility for melt flow, i.e. shrinkage and deformation between ZLS and the end of solidification results in porosity in the center area of slab.

The LMI model gives the opportunity to calculate that amount of volume which is not compensated by liquid filling between ZLS and the end of the mushy area, i.e. this will be the expected porosity level. The diagrams in Fig. 5 a/ show the pressure drop and the ZLS position for both roll settings in the casting case discussed above (see Fig. $3 \mathrm{a} /$ ). In this calculation $0.8 \mathrm{~mm}$ primary arm spacing and $5 \mathrm{mPas}$ melt viscosity were supposed.

In the case of constant roll gaps, the ferrostatic pressure reaches zero at $21.6 \mathrm{~m}$ (ZLS1) whereas in case of a prescribed setting it is $23.1 \mathrm{~m}$ (ZLS2). In the first case, the amount of necessary liquid flow is much larger (see Fig. 3a), which results in a greater pressure drop according to the Darcy-law.

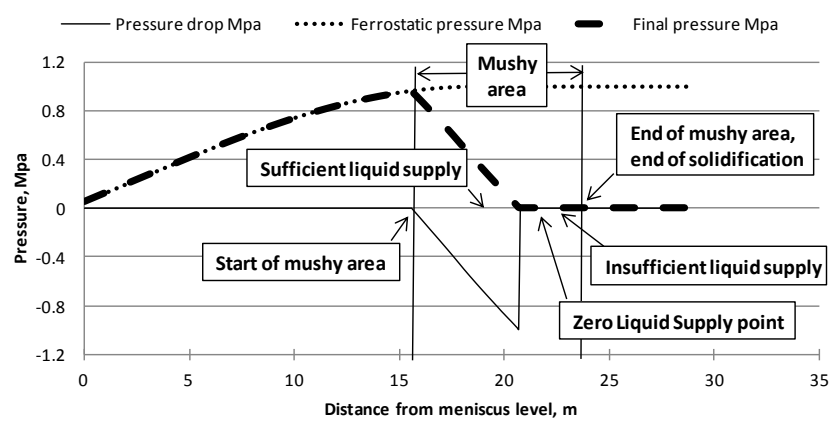

Figure 4

Pressure conditions in the mid part of slab

Between ZLS and the end of solidification zero ferrostatic pressure is assumed, which means that the cavities formed will not be filled by liquid melt. If the perfect and imperfect filling cases of these cavities are also calculated, the amount of cavities is characterized by the necessary liquid difference. These calculation results are displayed in Fig. 5b. Unit of porosity in the LMI model: $\mathrm{mm}^{2}$. 


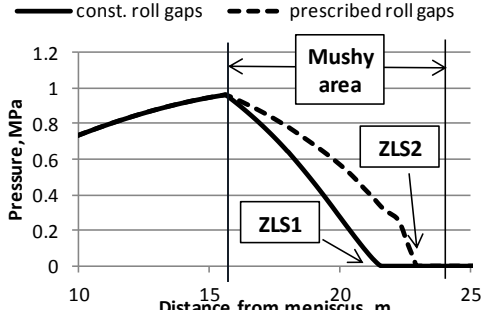

a/

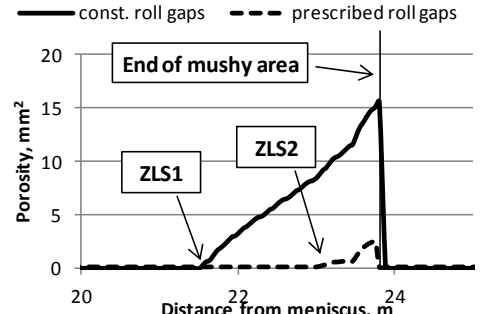

b/

Figure 5

ZLS positions (a/) and expected porosity values (b/) in the same casting case with two different roll settings

In the case of constant roll gaps the distance from ZLS1 to the end of solidification is $23.8-21.6=2.2 \mathrm{~m}$. If prescribed roll gaps are used, this value decreases to $23.8-23.1($ ZLS2) $=0.7 \mathrm{~m}$. The possibility of liquid supply is maintained for a much longer distance from meniscus in the case of prescribed roll gaps, and therefore the sum of discontinuities without melt filling will be much lower.

The expected porosity and centerline segregation level can be predicted by using the pressure drop and porosity functions.

\subsection{Effect of the Primary Structure on Pressure Drop and on Expected Porosity}

The permeability of the mushy area (Eq. (2-4)) is very sensitive to the solid dendrite primary structure that has developed. The finer the primary and secondary dendrite arm spacing, the lower the permeability. The diagrams in Fig. 6 show the effect of the primary structure on pressure drop process and on expected porosity level (same casting case as in Fig. 5, with prescribed roll gaps).

In Fig. 6 the primary arm spacing changes from 0.4 to $1.2 \mathrm{~mm}$. These values are realistic in the middle part of slabs cast under industrial casting conditions. The average value of primary spacing in the slab center is about $0.8-1.2 \mathrm{~mm}$ but a finer structure can be developed if tertiary arms are growing as primary arms or the composition changes because of enrichment. Improperly defined cooling rate and temperature gradient (distant from the surface of slab) can also result in structural parameters different from the average. Dendrites in Fig. 1 show a very fine structure despite the fact that they developed absolutely in the slab center, $120 \mathrm{~mm}$ away from the surface. Consequently, the primary structure has a great effect on the pressure drop process and on the possibilities of liquid feed. Dendrite arm spacing is also affected by several factors during casting. 


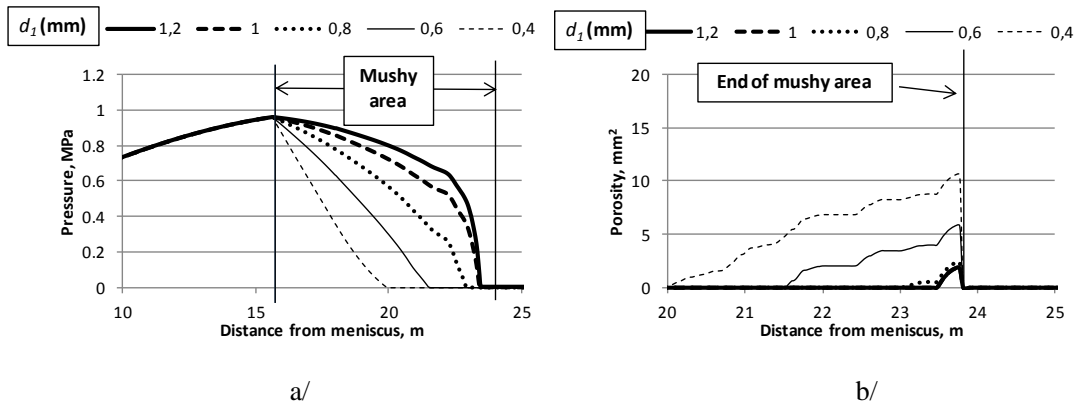

Figure 6

Effect of primary spacing on ferrostatic pressure drop in the mushy area (a/) and on expected centerline porosity (b/)

The local fluctuation of the primary dendrite structure around an average value can result in the variation of expected porosity and, accordingly, in the variation of macrosegregation and centerline segregation as well. We presume that this mechanism too plays a role in the variation of centerline segregation in the bridge formation mechanism and in the fluctuation of centerline segregation along the strand length. A detailed investigation of this problem including the effect of columnar to equiaxed transition is thus one of the directions of this research in the future.

At the end of solidification, the viscosity of the steel melt with a carbon content of $0.08-0.2 \%$ is around $5 \mathrm{mPas}$ according to calculations performed by JMatPro and IDS software. Only small variations of viscosity can be expected as a function of steel composition and liquid ratio, i.e. the position of the ZLS point is not significantly affected by the variation in viscosity.

\subsection{Estimation of the ZLS Position Based on a Statistical Analysis of Industrial Data}

The theory of porosity development outlined above is proven indirectly by an extended statistical analysis of a set of industrial data ${ }^{16}$. The analysis aimed to find a possible correlation between the complete technological database of slab castings and the centerline segregation index of cast products. The determination of centerline segregation index was based on an image analysis of porosity in the slab center. The highest level of correlation coefficient $(R)$ identified was very poor, only 0.469 . After the LMI model results were added to the analysis (i.e. shrinkage, deformation and roll gaps were also taken into account), the correlation coefficient increased significantly. In this calculation the position of the ZLS point (i.e. the position of the liquid supply stop) was defined at a given mushy liquid ratio (mushy liquid/complete mushy). Depending on the mushy liquid ratio, the correlation coefficient between measured and calculated porosity changed as 
shown in the diagram in Fig. 7. The maximum value of correlation coefficient was identified at a mushy liquid content of $30 \%$. This means that in industrial circumstances the blockage of liquid supply can be taken into account between $30 \%$ and $0 \%$ of mushy liquid ratio.

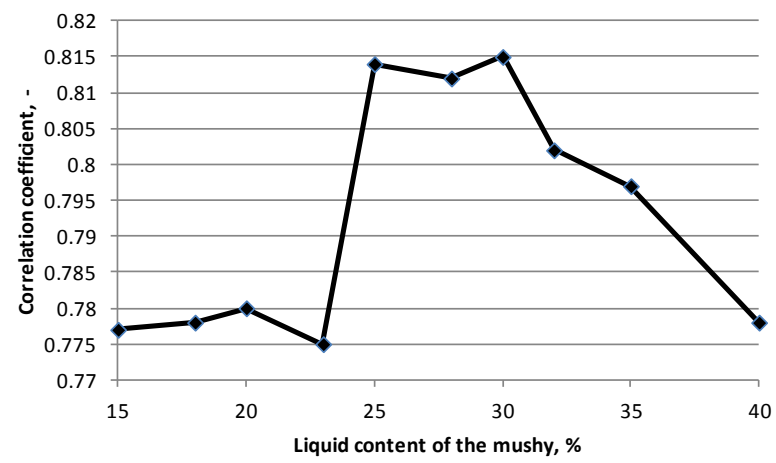

Figure 7

Change of correlation coefficient $(\mathrm{R})$ between measured and calculated porosities as a function of mushy liquid ratio from which the stoppage of liquid supply is supposed

At the beginning of the mushy area an average value of mushy liquid content of $60 \%$ can be assumed. Along the mushy area in the casting direction the mushy liquid decreases nearly linearly; the $30 \%$ of mushy liquid content can be found in approximately half of the mushy area. In the industrial application of the LMI model (presented in the following chapter) the calculation was performed with this assumption, i.e. the ZLS position was supposed to be at $30 \%$ of the mushy liquid content.

\section{Industrial Application}

\subsection{Introduction of the LMI Model}

The development, verification and application of the LMI model was performed in cooperation with ISD Dunaferr Co. Ltd. The company has two vertical slab casters (two strands per caster). The main objective of this project was to ensure enhanced slab quality from the technological side, to increase casting capacity and to add new steel grades and slab sizes to production. From the viewpoint of slab quality the most important aspect was: reducing the centerline segregation of slabs. 
ISD Dunaferr Co. Ltd. offers a wide range of products from the points of view of both steel grades and slab sizes. This is why, as a rule, the optimal setting of the supporting rolls is different in each case in order to ensure a centerline segregation level as low as possible. Due to the structural design of the casting machines, however, the roll gaps are fixed, and the same setting of supporting rolls is used in all casting cases. This fixed setting of rolls follows the shrinkage of the slab as a function of distance from the meniscus level.

A number of validation tests were performed in order to check the reliability of the model and of the calculated results. Numerous casting cases were modeled (thermal, solidification and LMI) and porosity results were evaluated on the basis of industrial experience. As a result, a proposal was made to modify the setting of the supporting rolls. According to preliminary calculations, the new setting was expected to produce a lower centerline segregation level for the complete diversified product structure (steel grades, slab sizes) of the steel plant.

\subsection{Introducing the New Setting of the Supporting Rolls}

On the basis of model calculation and validation tests, the management of ISD Dunaferr Co. Ltd. decided to modify the supporting roll setting according to the proposal.

The four strands were modified with utmost care, strand by strand. The process as a whole took about one year. During the conversion there were periods when the two strands of one casting machine were operated with different roll settings; in these periods we were able to evaluate the model amid particularly good conditions. In several casting cases the only difference between the two strands was the different setting of the roll gaps, thus the inner quality difference could directly be associated with the difference in roll gap setting.

The original and the modified roll settings are shown in Fig. 8 (the proposed change in the gap size affected the two last sections of rolls).

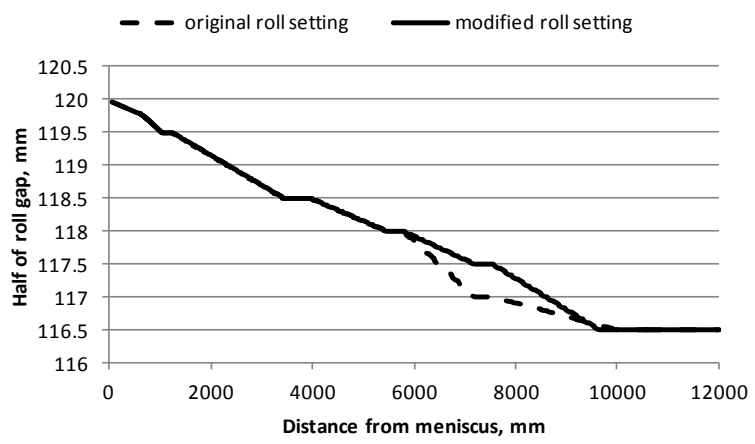

Figure 8

Original and modified settings of the supporting rolls 
In the transition period altogether 21 casting cases were investigated in detail. The cases were modeled. In order to control the centerline segregation level, the complete cross sections of the slabs were macroetched. Test results proved that the modified roll setting produce lower centerline segregation levels.

\section{Effect of the Modification of the Roll Gap}

\subsection{Investigation of a Particular Casting Case}

The effect of roll gap modification can be clearly identified in those casting cases where the two strands were operated with different roll gap settings (original and modified). In these cases steel composition, superheating, casting rates and cooling intensities are the same and the difference in inner qualities can be explained by the differing roll gaps. From the castings case No. 136 is discussed here. Steel composition and main casting parameters are shown in Table 1.

Table 1

Steel composition and main technological parameters of the cast slab

\begin{tabular}{|c|c|c|c|}
\hline \multicolumn{2}{|c|}{ Chemical composition } & \multicolumn{2}{|c|}{ Slab geometry (both strands) } \\
\hline $\mathrm{C}$ & $0.175 \mathrm{wt} \%$ & Thickness & $240 \mathrm{~mm}$ \\
\hline $\mathrm{Si}$ & $0.36 \mathrm{wt} \%$ & \multirow[t]{2}{*}{ Width } & $1360 \mathrm{~mm}$ \\
\hline $\mathrm{Mn}$ & $1.47 \mathrm{wt} \%$ & & \\
\hline S & $0.011 \mathrm{wt} \%$ & \multicolumn{2}{|c|}{ Supporting roll setting } \\
\hline $\mathrm{P}$ & $0.012 \mathrm{wt} \%$ & Strand No.1 & modified \\
\hline $\mathrm{Cr}$ & $0.078 \mathrm{wt} \%$ & \multirow[t]{2}{*}{ Strand No.2 } & original \\
\hline $\mathrm{Ni}$ & $0.036 \mathrm{wt} \%$ & & \\
\hline \multirow{3}{*}{ Superheating } & \multirow{3}{*}{$35^{\circ} \mathrm{C}$} & \multicolumn{2}{|c|}{ Casting rate } \\
\hline & & Strand No.1 & $0.53 \mathrm{~m} / \mathrm{min}$ \\
\hline & & Strand No.2 & $0.53 \mathrm{~m} / \mathrm{min}$ \\
\hline
\end{tabular}

Calculated porosity functions for the original and modified supporting roll settings can be seen in Fig. 9. The porosity developed in the last part of solidification was favorably affected by the modification of the roll gaps. On the basis of calculation results it was expected that the inner quality of the slab would improve. 


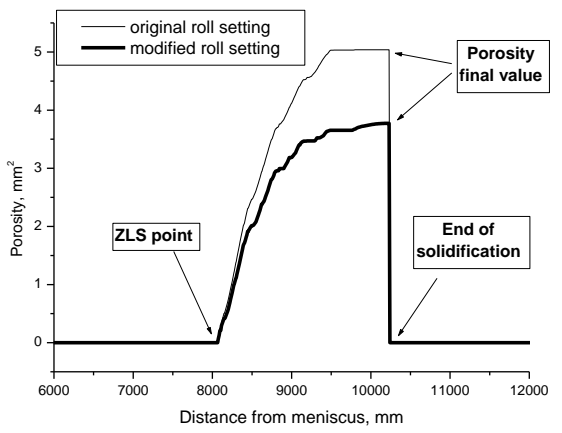

Figure 9

Expected porosity in casting case No. 136

In order to check the inner quality and the calculation results, samples were cut from both strands after casting. The samples (containing the whole cross sectional area) were ground and macroetched by ammonium-persulfate reagent. Pictures of macroetched samples can be seen in Fig. 10.

Before modification

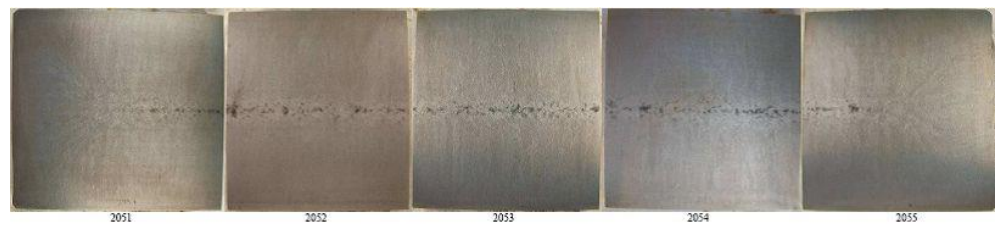

After modification

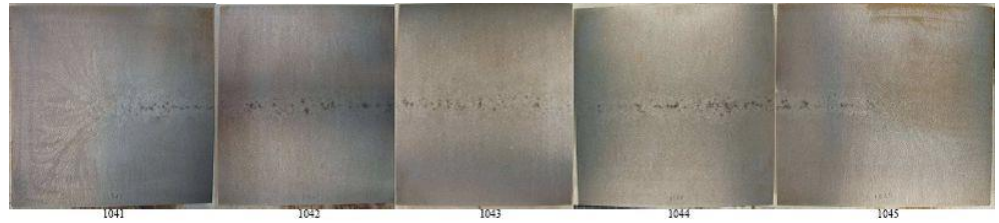

Figure 10

Macroetched cross sections of slabs cast with the original (above) and modified (bottom) roll setting (thickness of slab: $240 \mathrm{~mm}$ )

Describing the difference between the slabs in a numerical way is rather difficult. Yet, if the above pictures are compared, it can be clearly seen that the centerline segregation level is lower in the slab cast with the modified setting than in the slab cast with the original roll setting. After the modification, the segregated areas are smaller and the enriched parts are less coherent and more diffuse. At the same time, it can also be observed that the slab cast with the modified roll gaps contains several segregated areas. This means that the gap setting (in this particular case, at 
least) can be further refined. The diagram in Fig. 9 shows about $3.6 \mathrm{~mm}^{2}$ porosity in this case and, according to experience, centerline segregation cannot be identified in the cross section of slabs at a porosity of about $2.5 \mathrm{~mm}^{2}$.

\subsection{Overall Effect on Quality}

In order to get reliable feedback of the effects of the modified roll settings on the quality of cast products, ISD Dunaferr Co. Ltd. - Quality Department, conducted a detailed survey into customer evaluations (claims and rejections). Three periods were examined in the survey: one year before the modification of the roll setting (when all the slabs were cast with the original setting), one year during the modification of the gaps (when part of the slabs were cast with the original setting, part of them with the modified setting), and one year after modification (when all the slabs were cast with the modified roll gap setting). All customer claims and all rejects related to centerline segregation were analyzed, the sum of problematic shipments was calculated. The period before roll gap modification (one year) was chosen as reference (at 100\%). The results of survey can be seen in Fig. 11.

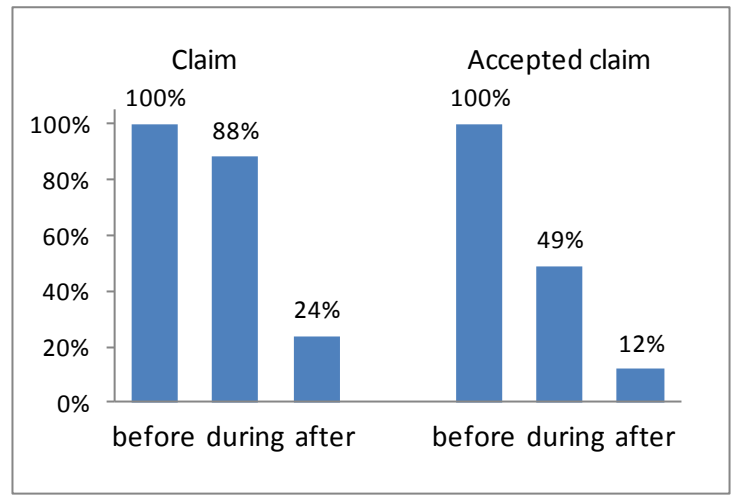

Figure 11

Customer claims and rejects related to centerline segregation before / during / after roll setting modification (each period of time represents one year)

The data above show a drastic decrease in quality problems related to centerline segregation. The number of claims decreased to $24 \%$. Regarding the number of investigated and accepted claims, there was almost a tenfold decrease.

\section{Summary}

Any decrease in the centerline segregation of continuously cast slabs requires a full harmony between steel composition, casting technology and casting machine settings, in particular in the last third of solidification. Centerline segregation can be avoided if the liquid, in equilibrium with the solid, does not move away from the vicinity of solid. Hence, in order to reach zero centerline segregation, the 
volumetric liquid flow rate function should be zero over the mushy area of slab. In practice, liquid moves in the slab because of volume changes. This flow is mainly generated by ferrostatic pressure developing within the strand.

The pressure drop calculation method and the LMI model can help answer the following important questions:

- How much liquid should move in the center of the slab in the mushy area, at a given steel composition, casting technology and casting machine settings?

- What level of porosity formation is likely to occur if flow and pressure drop occur?

If this model is applied, steel composition, casting technology and casting machine settings can be assessed in a complex way, especially from the point of view of centerline segregation formation.

If the LMI model is applied in practice, the position of the LMI point can be defined, on the basis of pressure drop calculations as well as of the statistical analysis of industrial data, at 30\% mushy liquid content. Below 30\% of mushy liquid, the dominant process is porosity formation, which can be affected by the sucking effect of cavities and by the pressure of released gases.

Based on the results of calculations aimed at defining the harmony between steel composition, casting technology and casting machine settings, ISD Dunaferr Co. Ltd. has changed the setting of supporting rolls on all its strands. Official data of the company's Quality Department, shows that the number of quality problems related to centerline segregation has decreased drastically due to the work presented in this paper.

\section{Acknowledgement}

We are very grateful for the financial contribution of EU Research Fund for Coal and Steel in the frame of Defect Free Casting (Deffree) project (RFSR-CT-200800007). The project was also supported by the assistance of the European Union, by the co-financing of the European Social Fund (TÁMOP-4.2.1.B-11/2/KMR2011-0001). The financial and technical support provided by ISD Dunaferr Co. Ltd. is especially highly appreciated.

\section{References}

[1] M. Réger M, B. Verő, Zs. Csepeli, Z. Szabó, R. Józsa and T. Kelemen: Mater Sci Forum, 729(2013) 175

[2] M. Réger, H. Kytönen, B. Verő and A. Szelig: Mater Sci Forum, 649(2010) 461

[3] DEFFREE - Integrated Models for Defect Free Casting, Final Report, RFSR-CT-2008-00007, 2012 
[4] M. Réger, H. Kytönen, B. Verö and A. Szelig: Mater Sci Forum, 589(2008) 43

[5] Y. Tsuchida, Y., Nakada, M., Sugawara, I., Miyahara, S., Murakami. and K. Tokushige: Transactions ISIJ, 24(1984) 899

[6] H. Jacobi: Steel Research, 74(2003) 667

[7] H. Presslinger, S. Ilie, P. Reisinger, A Schiefermüller, A. Pissenberger, E. Parteder and C. Bernhard.: ISIJ Int., 46(2006) 1845

[8] G. Lesoult: Mat. Sci. Eng. A, 413-414(2005) 19

[9] G. Krauss: Steels: Heat Treatment and Processing Principles, ASM Int. Metals Park Ohio, USA (1990) 6

[10] M. Réger, B.Verő, I. Kardos and P. Varga: Defect Diffus Forum, 297301(2010) 148

[11[ M. Réger, B. Verő, I. Kardos, E. R. Fábián and Gy. Kaptay: Mater Sci Forum, 659(2010) 441

[12] G. H. Geiger and D. R. Poirier: Transport Phenomena in Metallurgy, Addision-Wesley Publishing Company, Reading, MA (1973) 92

[13] M. S. Bhat, D. R. Poirier, and J. C. Heinrich: Metall. Mater. Trans. B, 26B(1995) 1049

[14] J. R. P. Rodrigues, M. M Mello, and R. G. Santos: Journal of Achievements in Materials and Manufacturing Engineering, 31(2008) 47

[15] Y. Natsume, D. Takahashi, K. Kawashima, E. Tanigawa and K. Ohsasa: ISIJ Int., 53(2013) 838

[16[ M. Réger, B. Verő, Zs. Csepeli and Á. Szélig: Mater Sci Forum, 508(2006) 233 